\title{
The Risk Elasticity Measure Index Exploration of Supply Chain and Mechanism Research
}

\author{
Ge Hong*
}

School of Business Administration, Nanchang Institute of Technology, Nanchang City, Jiangxi Province, 330099, China

\begin{abstract}
The modern supply chain were more vulnerable to risk, supply chain risk mainly comes from many aspects of the internal and external environment, the supply chain industry products background, upstream and downstream supply chain relationship, decision support. The study found that the need to establish information sharing mechanism, multilevel supply chain defense system, the principal-agent mechanism and supply chain emergency mechanism to build flexible supply chain, in order to achieve the purpose of supply chain risk management. In this paper, the establishment of a complete supply chain model with variable structure, through the simulation method for quantitative observation of supply chain flexibility curve, and abstract to describe the elastic curve of a set of parameters; after the design of simulation experiment to observe the different supply chain structure parameters (inventory level, supply chain, supplier selection for number of sources) influence supply chain resilience curve. At the same time to observe and analyze the supply chain by the recovery phenomena rules in the process of risk impact and elastic. And finally the quantitative impact evaluation of supply chain structure parameters on the supply chain flexibility.
\end{abstract}

Keywords: Information sharing, principal agent, supply chain, supply chain risk.

\section{INTRODUCTION}

In recent years, natural disasters, accidents, and other man-made interruption of supply chain risk issues concerned by people, and they bring to the supply chain and supply chain, damage increased with the length of the supply chain globalization, shortening of product lifecycles and unpredictable changes in market demand and the amplification. In order to deal with these difficult to overcome the high risk and low probability events, research on supply chain flexibility emerge as the times require [1]. Sheffi points out that increase supply chain flexibility means is to increase redundancy, improve supply chain flexibility, and the change of enterprise culture [2]. The key lies in the improvement of supply chain structure.

In the modern system of supply chain, supply chain related enterprises widely with lean production, global procurement, outsourcing, centralization strategy, supply chain to make itself more stable. But because of unexpected events in the supply chain operation process with the abruptness, complexity, destructive, persistent and chain characteristics such as a single on the above several aspects of supply chain management is not enough, the modern supply chain than ever more vulnerable to the impact of risk [3, 4].

Due to the subversion of the supply chain crisis nowadays more and more various, elastic in supply chain management plays an important role in the hitherto unknown [5]. So the industry leaders more efforts to seek the method to enhance supply chain flexibility. Therefore, supply chain flexibility knowledge system began to germinate. Supply chain flexibility not only means the ability to manage risk. Risk management now contains a deeper meaning: find yourself more suitable position in the supply chain disruption, even profit from [6]. Although we know various means can effectively improve the supply chain flexibility, but the effects of these methods for supply chain flexibility has no quantitative measure. For example: the increase in each node of the supply chain inventory levels and increasing the number of alternative suppliers can reduce the supply chain impact loss, but these two methods on the increase supply chain flexibility when each has what kind of rules, they are to enhance the supply chain flexibility effect on such as he, no quantitative description.

For quantitative appropriate method to describe the supply chain flexibility, and the influence law of supply chain structure parameters for the supply chain flexibility is observed by means of appropriate research methods, quantitative analysis of change law of supply chain flexibility in these parameters under the action of the quantitative evaluation of the supply chain, finally the elastic index is the purpose of this paper. Methods however want to find various methods for supply chain flexibility, the other is a prerequisite to the quantitative description and measurement of supply chain flexibility.

\section{RELATED THEORY}

\section{Overview of Research on Supply Chain Risk}

The types of supply chain risk can be divided into two kinds of operation risk and disruption risk. Operation risk 
refers to the internal factors of uncertainty, such as uncertain customer demand, uncertain supply, uncertain price etc. Disruption risk refers to a natural or man-made disaster such as earthquake, flood interruption, hurricanes or terrorist attacks, and similar floating exchange rates or strike economic crisis [7].

In order to mitigate supply chain risk, the company through cooperation mechanism with four basic steps: supplier management, demand management, production management and information management. Each measure is expected to improve supply chain operation through coordination and cooperation. First of all, the company ensured the flow of raw material supply chain efficiency. Second, the company can influence the demand change through the cooperation with downstream enterprises. Finally, if the company can get its partners all kinds of private information, the whole supply chain can achieve better coordination and cooperation [8].

\section{Overview of Research on Supply Chain Flexibility}

In materials science, flexibility refers to the ability to restore the prototype in the deformed material. In the corporate world, elasticity refers to the company by the ability after interruption recovery. The development of the company there are three main methods: elastic increase redundancy, establishing flexible and changing corporate culture. The utility of adding redundancy is limited, and the crucial role of flexibility and enterprise culture $[9,10]$.

In theory, the elastic enterprises can establish supply chain redundant increase. The organization can maintain a low inventory levels, production capacity of the higher utilization rate, at the same time have more suppliers. The company must pay more of the cost for inventory, production capacity and redundant workers; at the same time, such a mode of production makes the production and operation of more sluggish, reduce product quality, increase the cost of. In contrast, advanced production strategy currently respected such as Toyota production model, lean production process, the 6 Sigma production mode are committed to create high efficiency enterprise -- with few inventory to produce high quality products and timely logistics. In contrast, if the enterprises increase the flexibility, it can not only withstand severe disruption, and better able to respond to fluctuations in demand.

\section{Supply Chain Flexibility Curve}

A description of the enterprises in supply chain after the interruption performance recovery curve was shown in Fig. (1). Here the performance can refer to sales and production level, profits, customer service, inventory, order fulfillment rates, only an abstract index. The 8 phase diagrams are decided by the interruption of the characteristics and different stages of the supply chain reaction.

Step 1: Interrupt preparation. In some cases, the company can be foreseen and prepared to minimize disruption to a disruption of the damage. This warning can be Oklahoma GM company hurricane warning for 30 minutes, and can also be U. S. west coast port for months worsening workers' bargaining. In other cases, such as the 911 attacks and Philips fire, the warning is not exist.

Step 2: Stop start. That is when the hurricane, the accident occurred, the blasting effectiveness of terrorist attacks began, supplier, or any other high risk and low outage probability at the beginning of the.

Step 3: The first reaction. Interrupting the beginning, the main reaction (police, firefighters, front-line workers) began to pay attention to the original damage caused by interruption and respond. This time can be put out the fire in the time required to clean up the remains of the world trade center can also be needed for several months. The resources of enterprises will participate in the first reaction. In the interrupt other species, such as the strike and information technology results, the first reaction is to avoid physical damage and personal injury. Including the elimination of production operations to ensure that no workers or even in the case of information system, plant, equipment and personnel not subject to physical damage.

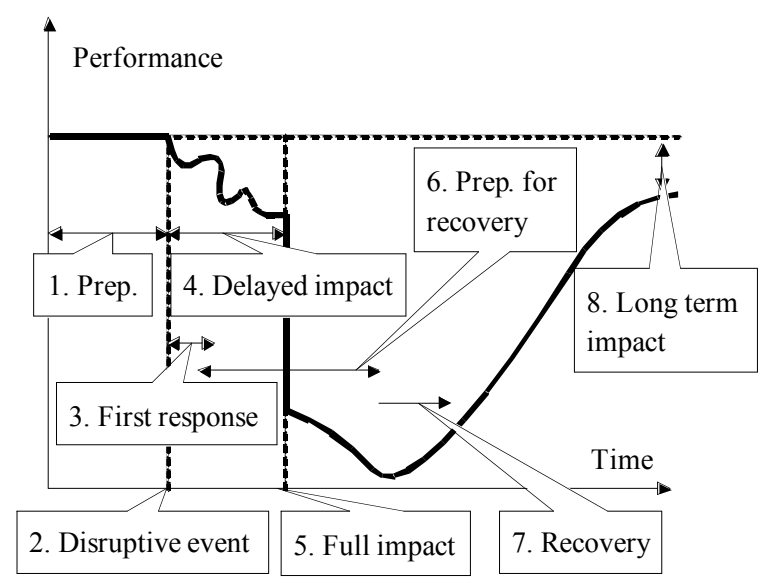

Fig. (1). A description of the enterprises in supply chain after the interruption performance recovery curve.

\section{THE MODEL OF SUPPLY CHAIN CONCEPT}

\section{The Three Layer Description}

According to the hierarchical SCOR model describes the supply chain concept, this paper will be divided into three layers: model description of the conception of supply chain logistics and information flow (Fig. 2), the realization mechanism of the operation mechanism of supply chain node specific tasks (described in the description of operation mechanism of various nodes).

The supply chain 24 hours of operation, which is composed of four kinds of nodes: customers, retailers, manufacturers, assemblers. The customer node a, node a retailer, assemblers of arbitrary random nodes, node level any manufacturer. Interaction of logistics and information flow between the customer and the retailers, the interaction of logistics and information flow of retailers and any adjacent assemblers, assemblers directly connected arbitrary a level assemblers or manufacturer interaction of logistics and information flow. Series of supply chain can through the series regulator assemblers adjustment is greater than or equal to any level 3. 


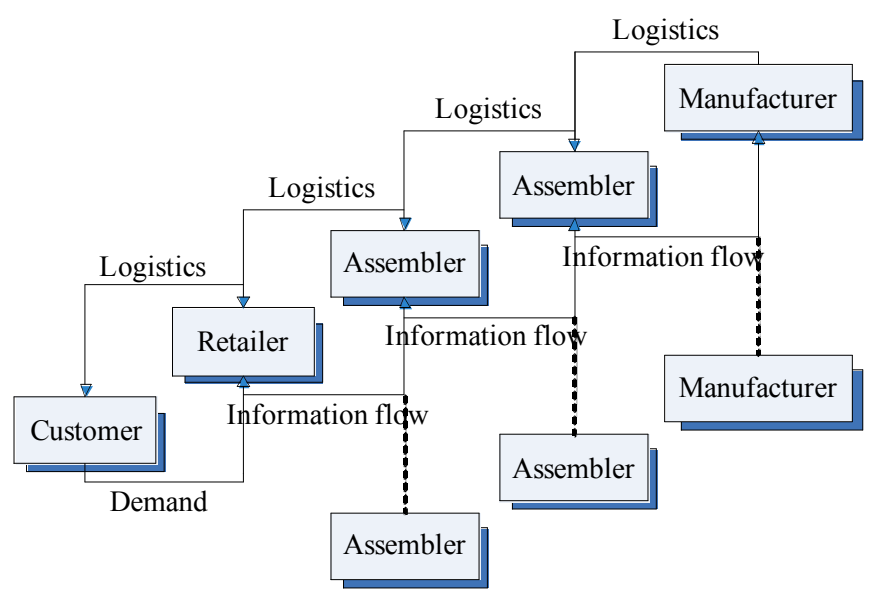

Fig. (2). Model description of the conception of supply chain logistics and information flow.

Retailers use static $\left(S_{R}, S_{R}\right)$ strategy inventory and order management decision. Assuming that $O_{R}$ is the size of the order, $I_{R}$ when the inventory level, $I_{\text {expected }}$ is issued and unfulfilled orders, $I_{b a c k \log }$ is under the lower customer cargo, then

$$
\begin{gathered}
O_{R}= \begin{cases}S_{R}-I_{R}^{\prime} & \text { if } I_{R}^{\prime}<s_{R} \\
0 & \text { if } I_{R}^{\prime}<s_{R}\end{cases} \\
I_{R}{ }^{\prime}=I_{R}+I_{\text {expected }}-I_{\text {back } \log }
\end{gathered}
$$

Assuming that $I_{R}(t)$ is a time $\mathrm{t}$ inventory of water goods retailer, $I_{R}(t)$ can be positive, negative or 0 . Then $I_{R}^{+}(t)=\max \left\{I_{R}(t), 0\right\}$ is $\mathrm{t}$ times the actual inventory level is non negative. $I_{R}^{-}(t)=\max \left\{-I_{R}(t), 0\right\} \mathrm{t}$ time retailers did not complete the order quantity, is non negative. After $n$ days of operation, the average daily inventory levels for retailers

$\bar{I}_{R}^{+}=\frac{\int_{0}^{n} I_{R}^{+}(t) d t}{n}$

The retailer's average daily unfinished orders:

$\bar{I}_{R}^{-}=\frac{\int_{0}^{n} I_{R}^{-}(t) d t}{n}$

\section{Assembler Operation Mechanism}

Assuming that the assembly each finished piece need time hours, each production preparation time is 1 hours. Considering the assembly time can not be more than a day (second days will start generating assembly new), it is ultimately the implementation of assembly part number A:

$$
A= \begin{cases}\min \left(A_{A}, I_{P}\right) & \text { if } \min \left(A_{A}, I_{P}\right) \times R_{A} \leq 23 \\ \frac{23}{R_{A}} & \text { if } \min \left(A_{A}, I_{P}\right) \times R_{A}>23\end{cases}
$$

Finally the implementation of assembly time $T_{A}$ (hours) for

$T_{A}= \begin{cases}1+\min \left(A_{A}, I_{P}\right) \times R_{A} & \text { if } \min \left(A_{A}, I_{P}\right) \times R_{A} \leq 23 \\ 24 & \text { if } \min \left(A_{A}, I_{P}\right) \times R_{A}>23\end{cases}$

After assembly work began performing assembly business check inventory of raw materials, the decision will be the best supplier of raw materials to set the number of $O_{P}$. $I_{\text {expected }}$ is issued and unfulfilled orders (i.e. stock in transit), $I_{\text {backlog }}$ is under lower customer cargo volume. The decision will be assembly products quantity e assemblers using static $\left(s_{P}, S_{P}\right)$ strategy:

$O_{P}= \begin{cases}S_{P}-I_{P}^{\prime} & \text { if } I_{P}^{\prime}<S_{P} \\ 0 & \text { if } I_{P}^{\prime}<S_{P}\end{cases}$

$I_{P}^{\prime}=I_{P}+I_{\text {expected }}-I_{\text {back } \log }$

Initial stock finally assume that assemblers of $I_{A}(0)=I_{P}(0)=80$, and the initial conditions did not complete the order. Assembler at time point $t=0,1, \ldots, n-1$. check inventory.

\section{The Manufacturer}

Manufacturers play terminal vendors here role. Supply chain is also produced original materials here.

As shown in Fig. (3), in the daily 00:00, the manufacturer first check whether the assembly's unfinished orders, including orders may have just arrived. If there is unfinished orders and inventory sufficient to meet one or more of the entire order, the manufacturer will accord to the FIFO sequence is issued to one or more integer order goods. This part of the order fulfillment is not allowed. While the transport time is a random variable, subject to the normal $(0.2,1)$ day distribution, 1 days is 0.2 mean, variance. The new inventory for the old product inventory minus the amount issued amount of goods. Haven't been completed orders in order to save unfinished record.

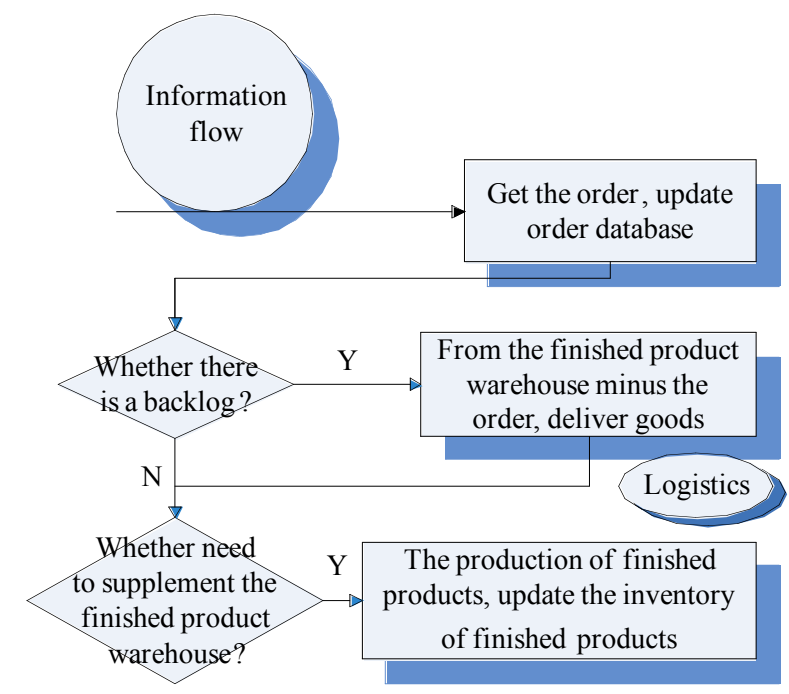

Fig. (3). Manufacturers operating mechanism. 
In sending goods to assemblers, manufacturers to check the finished goods inventory $I_{F}, I_{b a c k \log }$ is under lower customer cargo, then decided to create the number of finished $M_{F}$. Assuming that the manufacture material. The decision will be assembly products quantity $\left(s_{F}, S_{F}\right)$ assemblers using static $M_{F}$ strategy:

$$
\begin{aligned}
& M_{F}= \begin{cases}S_{F}-I_{F}^{\prime} & \text { if } I_{F}^{\prime}<S_{F} \\
0 & \text { if } I_{F}^{\prime}<S_{F}\end{cases} \\
& I_{F}{ }^{\prime}=I_{F}-I_{b a c k \log }
\end{aligned}
$$

Assuming that the manufacture each finished piece need time hours, every manufacturing job preparation time of 1 hours. Considering the manufacturing time is no more than a day (second days beginning will produce new manufacturing operations), is the ultimate implementation of manufactured product number $\mathrm{M}$ :

$M= \begin{cases}M_{F} & \text { if } M_{F} \times R_{F} \leq 23 \\ \frac{23}{R_{F}} & \text { if } M_{F} \times R_{F}>23\end{cases}$

Finally the implementation of manufacturing time (hours) for:

$T_{F}= \begin{cases}1+M_{F} \times R_{F} & \text { if } M_{F} \times R_{F} \leq 23 \\ 24 & \text { if } M_{F} \times R_{F}>23_{P}\end{cases}$

\section{Supply Chain Information Sharing Mechanism}

The core enterprise in supply chain information sharing mechanism of the core enterprise based on refers to, is at the core position play a leading role in the supply chain alliance, in logistics, information flow, capital flow, technology, management coordination, strength is very strong and cohesive enterprise, around the core enterprise construction supply chain, not just from a supply and demand the supplier's supplier to the customer the customer simply

The value of existence of the core enterprise is that it is the promoter and ensure the efficient operation of the supply chain, it can make the whole supply chain always keep improving the pressure strong, how its function effect, directly affect the coordination and the efficiency of the supply chain. Construction as shown in Fig. (4) of supply chain information sharing mechanism based on the core business.

Design of the management information system of the core enterprise is the guarantee of the whole supply chain system information in a timely manner to eliminate the bullwhip effect, smooth transmission, the most important and complex work to realize sharing information process, the node design of enterprise management information system is relatively simple. On the core enterprises, establish the sharing mechanism of the core enterprise information based on core enterprise management information integrated system can be managed system of node enterprises and their own, centralized management can realize the information resource sharing, to achieve full information.

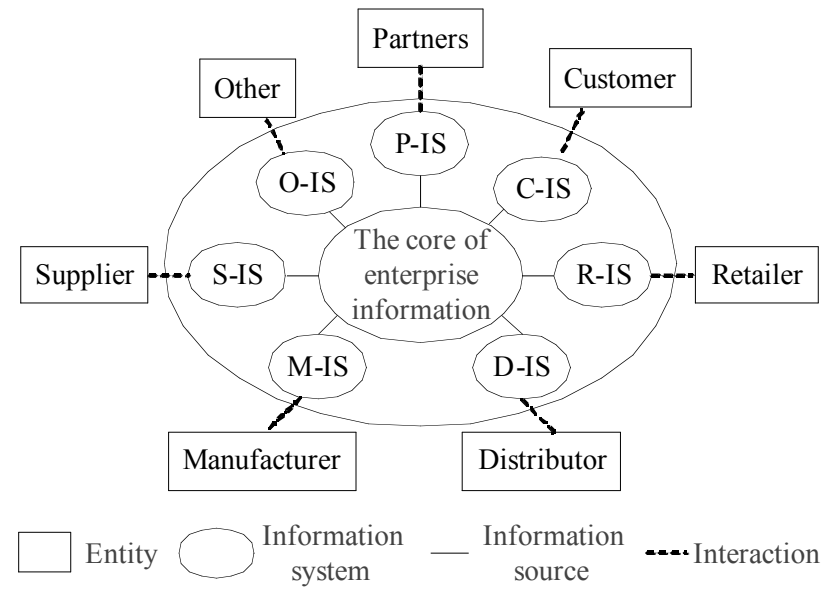

Fig. (4). Construction in of supply chain information sharing mechanism.

\section{EXPERIMENTAL RESULTS}

\section{The Model Structure}

Retailer stands for retailers, Wholesaler represents the assemblers, Factory represents the manufacturers. Each object class are independent units, able to make independent decisions, and object associated with the existence of clear interface. Thus the use of these objects can evolve arbitrarily complex supply chain network.

In order to be able to through the combination of object graphic connection to build any structure of the supply chain network, in the model using the object interface (Anylogic ports) as the interface between objects. Easily through the click of a mouse can be connected to Ports.

As shown in Fig. (5), the retailer and the assembly are each have two interfaces: one for receiving orders and send the finished product, one to send the order and receipt of raw materials. The manufacturer is only one interface, because we assume that the initial raw material is infinite, the supply chain node before the manufacturer shall not be considered.

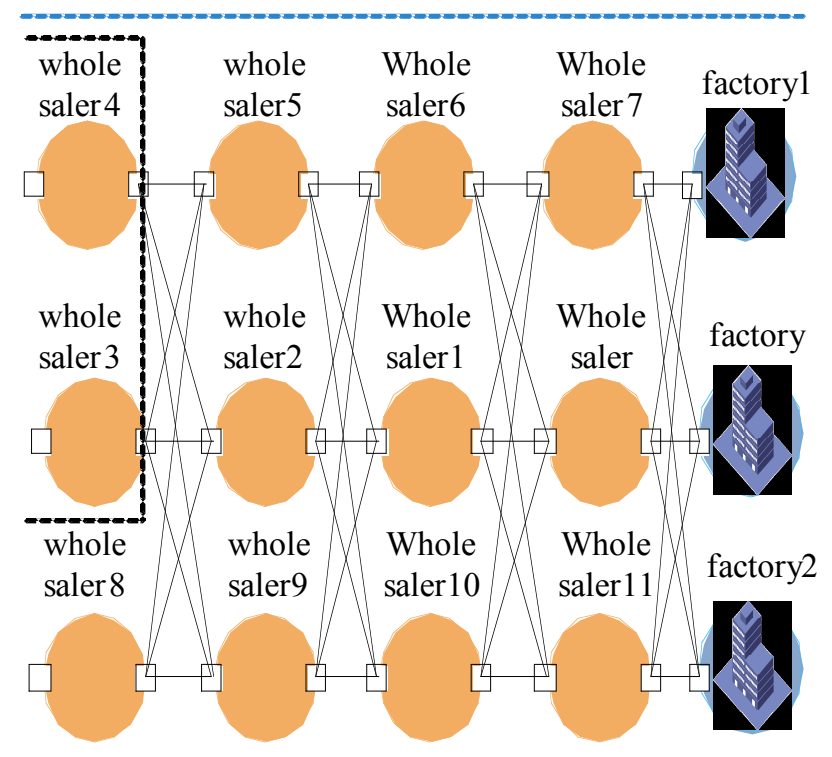

Fig. (5). The top structure of supply chain model. 
The reason for creating object class customers not in Anylogic is because customers in addition to generate demand and statistical demand waiting time no other complex behavior. The generation and statistical requirements can use event generator in a top model (event generation) easily.

\section{Data Acquisition}

Running is obtained after the typical supply chain inventory curve, prove that the model developed successfully (Fig. 6).

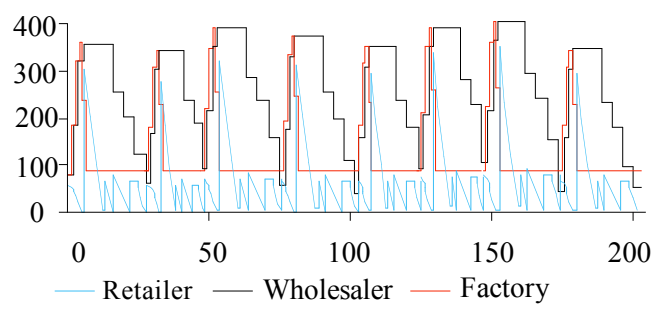

Fig. (6). The obtained typical supply chain inventory curve.

In order to various risk environment simulation of the supply chain, need to change the definition of various parameters in the program. For example, the requirement of simulation mutation (Fig. 7), you can define the customer demand rate as a function of time variation; mutation simulated production capacity, will be a function definition manufacturers productivity change over time.

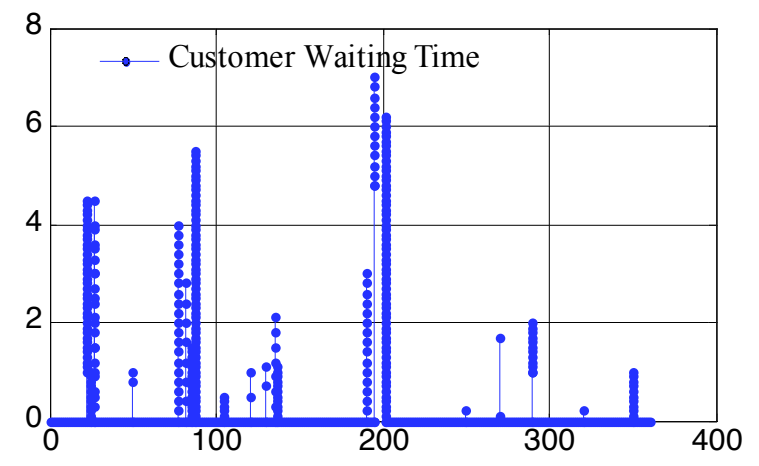

Fig. (7). Customer waiting time curve.

\section{Simulation of Elastic Curve}

The purpose of this experiment is to quantitatively analyze the influence of parameter of supply chain structure for the elasticity. Parameter of supply chain structure is easy to describe, and supply chain flexibility is characteristic of a multidimensional description, it is the best method in elastic curve. However, the quantitative relationship between the structure parameters and a representation of a curve is not easy. The best way is to describe a curve with a set of parameters, find the function relationship between these parameters in each individual parameter and supply chain structure parameters, in order to characterize the relationship between supply chain structure parameters and elastic curve.

Before the simulation experiments are carried out (Fig. 8), the first 10 test run to observe the elastic curve of possible situations. According to the elastic characteristic curve of concrete, custom used to describe the elastic curve of a set of parameters. Such as increasing the elastic curve in the case of customer demand process are in line with the four stages of figure 234: normal operation period (before 1), the main effect of amplifying futures influence period (1 to 2); impact of weakening or residual influence period (2 to 3 ), restore the normal operation period (after 3).

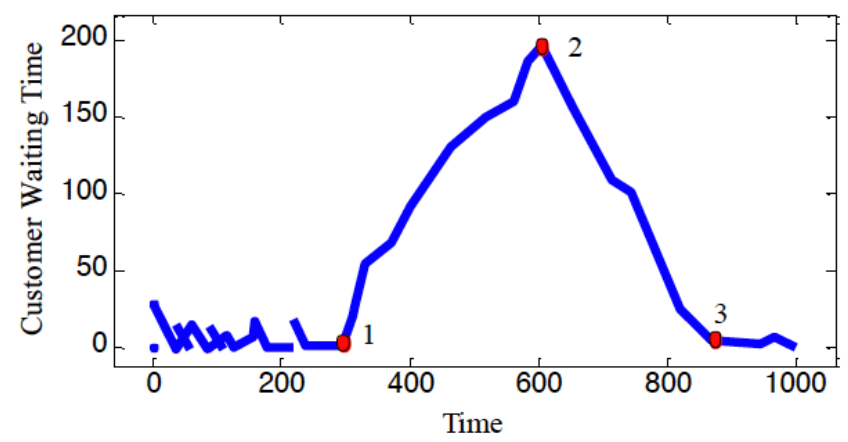

Fig. (8). Sudden demand elastic recovery curve.

Through the observation can be found in the following set of parameters accurately and comprehensively describe the elastic curve: risk before the influence of average waiting time, the main effect of the average waiting time, the residual effect of the average waiting time, the average waiting time for after effects, the main effect of waiting time amplification, the residual impact of waiting time amplification, residual effects of duration.

The influence of the risk before the average waiting time, the main effect of the average waiting time, the residual effects of the average waiting time, average waiting time after the effect of the four parameters to describe the performance of supply chain is the change in absolute volume. The main effect of waiting time amplification, residual effect of waiting time amplification of these two parameters are used to describe the supply chain performance for interrupt sensitivity.

Another set of parameters are obtained to describe the elastic curve of supply chain supply capacity of anticlimax can be used the same method: risk before the influence of average waiting time, side effect of the average waiting time, the main effect of the average waiting time, the average waiting time for after effects, side effects of waiting time amplification, the main effect of waiting time amplification, side effects start time lag, the main effect of start time lag, lag time end effect.

The output file into the MINITAB statistics software rendered the customer waiting time column chart (Fig. 9). The abscissa is the demand generated time, longitudinal coordinates for the same time period (interval of histogram) all demand generated by the waiting time.

It can be seen in the time period $(300,600)$ column increased rapidly. This is because the demand in the period of time the arrival rate increased by 10 times, due to not adapt to the supply chain supply capacity, the waiting time of each demand increasing. In 600 days, a sudden drop in a column chart. This is because the demand arrival rate reduced to $1 / 10$ of the original, and the supply chain supply ability recover gradually adapt to, the waiting time of each demand gradually returned to normal level. 


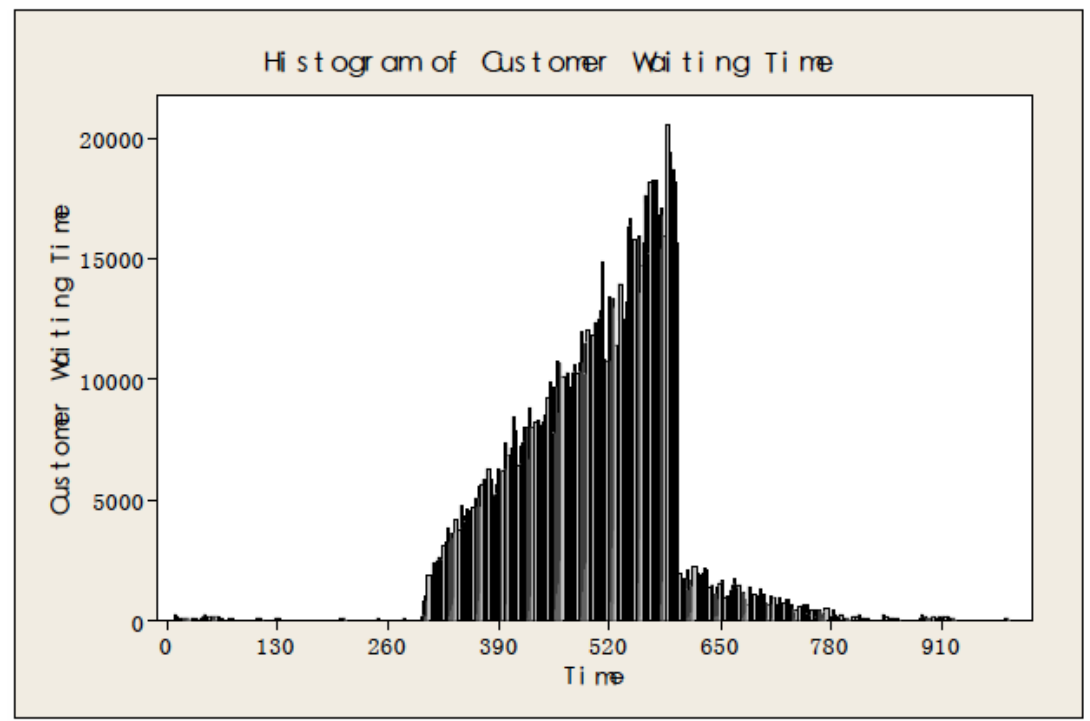

Fig. (9). The output file into the MINITAB statistics software rendered the customer.

\section{CONCLUSION}

This paper arise from the practical problems in production, carries on the simulation experiment of supply chain resilience curve obtained by simulation and. Thus put forward a set of method to quantitative parameters describing the supply chain flexibility. On this basis through the simulationexperiment and data analysis, we obtain the relationship between supply chain structure parameters and supply chain flexibility. Put forward the constructive suggestion to the enterprises.

\section{CONFLICT OF INTEREST}

The author confirms that this article content has no conflict of interest.

\section{ACKNOWLEDGEMENTS}

Declared none.

\section{REFERENCES}

[1] J. B. Barney, "Purchasing, supply chain management and sustained competitive advantage: The relevance of resource - based theory," Journal of Supply Chain Management, vol. 48, no. 2, pp. 3-6, 2012.

[2] C. Shepherd, and H. Günter, "Measuring supply chain performance: current research and future directions," Behavioral Operations in
Planning and Scheduling, Springer Berlin Heidelberg, pp. 105-121, 2011.

[3] S. Apte, and B. Carlin, "Inclusion of relevant tests in the Pharmacopoeia to improve supply chain integrity," Journal of Excipients and Food Chemicals, vol. 4, no. 3, pp. 61-63, 2013.

[4] W. S. Boyce, "Supply Chain Relationships in Procurement: Is Collaboration Reality?," University Of Missouri-Saint Louis, 2014.

[5] D. Turker, and C. Altuntas, "Sustainable supply chain management in the fast fashion industry: An analysis of corporate reports," European Management Journal, vol. 32, no. 5, pp. 837-849, 2014.

[6] H. Y. Ching, and M. A. Moreira, "Management Systems and Good Practices Related to the Sustainable Supply Chain Management," Journal of Management and Sustainability, vol. 4, no. 2, pp. 34, 2014.

[7] R. Baldwin, and J. Lopez - Gonzalez, "Supply - chain Trade: A Portrait of Global Patterns and Several Testable Hypotheses," The World Economy, 2014.

[8] P. J. Daugherty, A. E. Ellinger, and M. B. Myers, "Automatic Replenishment Programs: Forging Supply Chain Relationships," In: Proceedings of the 1999 Academy of Marketing Science (AMS) Annual Conference. Springer International Publishing, pp. 219-219, 2015.

[9] A. W. Mackelprang, J. L. Robinson, E. Bernardes, and G. S. Webb, "The Relationship Between Strategic Supply Chain Integration and Performance: A Meta - Analytic Evaluation and Implications for Supply Chain Management Research," Journal of Business Logistics, vol. 35, no. 1, pp. 71-96, 2014.

[10] J. Jayaram, M. Dixit, and J. Motwani, "Supply chain management capability of small and medium sized family businesses in India: A multiple case study approach," International Journal of Production Economics, vol. 147, pp. 472-485, 2014.

Received: June 10, 2015

(C) Ge Hong; Licensee Bentham Open.

This is an open access article licensed under the terms of the (https://creativecommons.org/licenses/by/4.0/legalcode), which permits unrestricted, noncommercial use, distribution and reproduction in any medium, provided the work is properly cited. 